\title{
Molecular mechanism of self-antigen recognition by the ligand binding domain of $B$ cell inhibitory co-receptor CD72
}

\author{
N. Numoto ${ }^{1}$, K. Hirata ${ }^{2}$, C. Akatsu ${ }^{3}$, T. Tsubata ${ }^{3}$, N. Ito ${ }^{1}$ \\ ${ }^{1}$ Department of Structural Biology, Medical Research Institute, Tokyo Medical and Dental University (TMDU), 1-5-45 Yushima \\ Bunkyo-ku, Tokyo 113-8510, JAPAN, ${ }^{2}$ RIKEN SPring-8 Center, 1-1-1 Kouto, Sayo-cho, Sayo-gun, Hyogo 679-5148, JAPAN, \\ ${ }^{3}$ Department of Immunology, Medical Research Institute, Tokyo Medical and Dental University (TMDU), 1-5-45 Yushima Bunkyo-ku, \\ Tokyo 113-8510, JAPAN \\ numoto.str@mri.tmd.ac.jp
}

CD72 is an inhibitory co-receptor that negatively regulates B cell antigen receptor (BCR) signalling. The ligand-binding domain of CD72 at the extracellular region belongs to the C-type lectin-like domain (CTLD) superfamily. We have demonstrated that it recognizes the nuclear autoantigen Sm/RNP composed of proteins and RNA, and suppresses autoimmune diseases such as systemic lupus erythematosus [1]. The crystal structure of the ligand-binding domain of mouse CD72a , a lupus-resistant allele, has been determined at $1.2 \AA$ resolution. Electrostatic potential analysis of the molecular surface of CD72 ${ }^{\mathrm{a}}$-CTLD suggest that charge distribution at the putative ligand-binding site may affect the binding affinity between CD72 and Sm/RNP.

We have determined the crystal structure of the ligand-binding domain of mouse $\mathrm{CD} 72^{\mathrm{c}}$, a lupus-susceptible allele with reduced affinity to Sm/RNP. The obtained crystals were large enough for X-ray diffraction experiments of about $200 \mu \mathrm{m}$ cubic, but clusters of hundreds or thousands of microcrystals (Fig. 1). Development of the micro focus X-ray beam and rapid automated data collection [2] and processing [3] systems at SPring-8 enabled us to obtain a full data set that allowed the successful structure determination and refinement at $2.5 \AA$ resolution (Fig. 2). We took 1,400 of small-wedge (10 degree) data from 14 crystals. The data were classified based on the unit-cell dimensions or correlation coefficient between data and merged to a full data set for structure determination. Analysis of the hierarchical clustering of the small-wedge data shows that the crystal packing varies along with the $c$-axis direction, but no significant conformational variations were observed among the crystal structures.

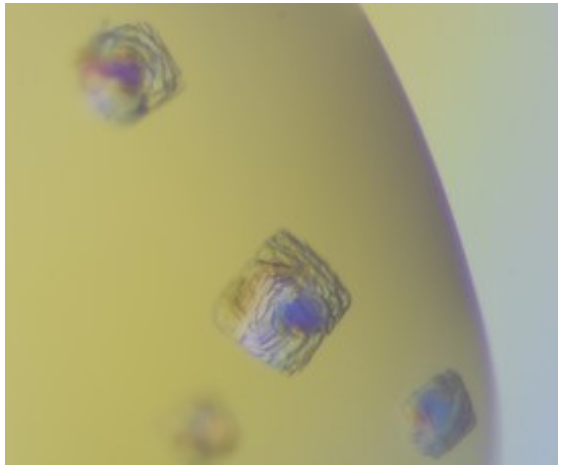

Figure 1. Crystals of CD72 ${ }^{\mathrm{c}}-\mathrm{CTLD}$

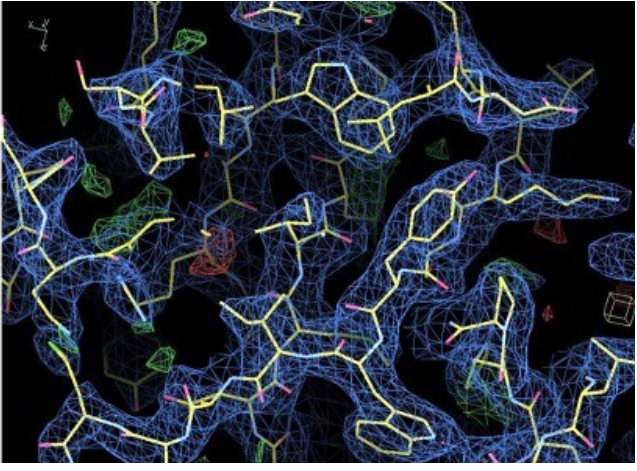

Figure 2. Electron density map of CD72 - CTLD

The obtained structure reveals that substitutions of amino acids at the ligand-binding site do cause the inversion of the charge distribution of the molecular surface as we hypothesized. Charge repulsion between CD72 ${ }^{\mathrm{c}}$-CTLD and strong negative charges of RNA of Sm/RNP would be the molecular mechanism of reduced affinity.

[1] Akatsu, C., Shinagawa, K., Numoto, N., Liu, Z., Ucar, A. K., Aslam, M., Phoon, S., Adachi, T., Furukawa, K., It,o N. \& Tsubata, T. (2016) J. Exp. Med. 213, 2691.

[2] Hirata, K., Yamashita, K., Ueno, G., Kawano, Y., Hasegawa, K., Kumasaka, T. \& Yamamoto M. (2019) Acta Cryst. D75, 138.

[3] Yamashita, K., Hirata, K. \& Yamamoto, M. (2018) Acta Cryst. D74, 441.

Keywords: crystal structure; immune system; nucleic acid 